\title{
Ataxia, Dementia, and Hypogonadotropism Caused by Disordered Ubiquitination
}

\section{Citation}

Margolin, David H., Maria Kousi, Yee-Ming Chan, Elaine T. Lim, Jeremy D. Schmahmann, Marios Hadjivassiliou, Janet E. Hall, et al. 2013. "Ataxia, Dementia, and Hypogonadotropism Caused by Disordered Ubiquitination." New England Journal of Medicine 368 (21) (May 23): 1992-2003. doi:10.1056/nejmoa1215993.

\section{Published Version}

10.1056/nejmoa1215993

\section{Permanent link}

http://nrs.harvard.edu/urn-3:HUL.InstRepos:32697820

\section{Terms of Use}

This article was downloaded from Harvard University's DASH repository, and is made available under the terms and conditions applicable to Other Posted Material, as set forth at http:// nrs.harvard.edu/urn-3:HUL.InstRepos:dash.current.terms-of-use\#LAA

\section{Share Your Story}

The Harvard community has made this article openly available.

Please share how this access benefits you. Submit a story.

\section{Accessibility}




\title{
Ataxia, Dementia, and Hypogonadotropism Caused by Disordered Ubiquitination
}

\author{
David H. Margolin, M.D., Ph.D., Maria Kousi, Ph.D., \\ Yee-Ming Chan, M.D., Ph.D., Elaine T. Lim, M.S., Jeremy D. Schmahmann, M.D., \\ Marios Hadjivassiliou, M.D., Janet E. Hall, M.D., Ibrahim Adam, M.D., \\ Andrew Dwyer, N.P., Lacey Plummer, B.S., Stephanie V. Aldrin, B.A., \\ Julia O’Rourke, Ph.D., Andrew Kirby, B.S., Kasper Lage, Ph.D., \\ Aubrey Milunsky, M.B., B.Ch., D.Sc., Jeff M. Milunsky, M.D., \\ Jennifer Chan, M.D., E. Tessa Hedley-Whyte, M.D., Mark J. Daly, Ph.D., \\ Nicholas Katsanis, Ph.D., and Stephanie B. Seminara, M.D.
}

From the Department of Neurology (D.H.M., J.D.S.), Harvard Reproductive Sciences Center and Reproductive Endocrine Unit (Y.-M.C., J.E.H., A.D., L.P., S.V.A., J.O., S.B.S.), Analytic and Translational Genetics Unit (E.T.L., A.K., K.L., M.J.D.), Department of Medicine, Pediatric Surgical Research Laboratories (K.L.), and Department of Neuropathology (E.T.H.-W.), Massachusetts General Hospital, Division of Endocrinology, Department of Medicine, Boston Children's Hospital (Y.-M.C.), and Department of Pathology, Brigham and Women's Hospital (J.C.) - all in Boston; Center for Human Genetics, Cambridge, MA (A.M., J.M.M.); Center for Human Disease Modeling, Department of Cell Biology (M.K., N.K.), and Department of Pediatrics (N.K.), Duke University Medical Center, Durham, NC; Department of Neurology, Royal Hallamshire Hospital, Sheffield, United Kingdom (M.H.); Specialty Hospital, Amman, Jordan (I.A.); and Center for Biological Sequence Analysis, Technical University of Denmark, Lyngby, and Center for Protein Research, University of Copenhagen, Copenhagen (K.L.). Address reprint requests to $\mathrm{Dr}$. Seminara at the Reproductive Endocrine Unit, Massachusetts General Hospital, Boston, MA 02115, or at seminara.stephanie@ mgh.harvard.edu; or to Dr. Katsanis at the Center for Human Disease Modeling, Duke University, Durham NC 27710, or at katsanis@cellbio.duke.edu.

Drs. Margolin, Kousi, and Y.-M. Chan and Drs. Katsanis and Seminara contributed equally to this article.

This article was published on May 8, 2013, at NEJM.org.

N Engl J Med 2013;368:1992-2003.

DOI: 10.1056/NEJMoal215993

Copyright () 2013 Massachusetts Medical Society.
ABSTRACT

\section{BACKGROUND}

The combination of ataxia and hypogonadism was first described more than a century ago, but its genetic basis has remained elusive.

\section{METHODS}

We performed whole-exome sequencing in a patient with ataxia and hypogonadotropic hypogonadism, followed by targeted sequencing of candidate genes in similarly affected patients. Neurologic and reproductive endocrine phenotypes were characterized in detail. The effects of sequence variants and the presence of an epistatic interaction were tested in a zebrafish model.

\section{RESULTS}

Digenic homozygous mutations in RNF216 and OTUD4, which encode a ubiquitin E3 ligase and a deubiquitinase, respectively, were found in three affected siblings in a consanguineous family. Additional screening identified compound heterozygous truncating mutations in RNF216 in an unrelated patient and single heterozygous deleterious mutations in four other patients. Knockdown of inf216 or otud4 in zebrafish embryos induced defects in the eye, optic tectum, and cerebellum; combinatorial suppression of both genes exacerbated these phenotypes, which were rescued by nonmutant, but not mutant, human RNF216 or OTUD4 messenger RNA. All patients had progressive ataxia and dementia. Neuronal loss was observed in cerebellar pathways and the hippocampus; surviving hippocampal neurons contained ubiquitin-immunoreactive intranuclear inclusions. Defects were detected at the hypothalamic and pituitary levels of the reproductive endocrine axis.

\section{CONCLUSIONS}

The syndrome of hypogonadotropic hypogonadism, ataxia, and dementia can be caused by inactivating mutations in RNF216 or by the combination of mutations in RNF216 and OTUD4. These findings link disordered ubiquitination to neurodegeneration and reproductive dysfunction and highlight the power of whole-exome sequencing in combination with functional studies to unveil genetic interactions that cause disease. (Funded by the National Institutes of Health and others.) 
I

N RECENT YEARS, WE HAVE SEEN GREAT ADvances in the elucidation of genetic causes of cerebellar ataxia, with newly identified genes regulating a wide spectrum of cellular functions, including intracellular signaling, tau regulation, and mitochondrial function. ${ }^{1}$ However, a genetic defect cannot be found in approximately $40 \%$ of patients with ataxia, ${ }^{1}$ including those in whom ataxia is associated with reproductive endocrine failure, a syndrome first reported by Gordon Holmes in $1908 .^{2}$ Most patients with this syndrome have a hypogonadotropic condition, with defective secretion of gonadotropins by the pituitary gland. ${ }^{3-12}$ Strikingly, genes associated with ataxia have little functional overlap with genes associated with hypogonadotropic hypogonadism, which encode proteins involved in the biologic function of the neurons that secrete gonadotropinreleasing hormone $(\mathrm{GnRH}) .^{13}$

A decade ago, we described a consanguineous family with a syndrome of cerebellar ataxia, dementia, and hypogonadotropic hypogonadism. ${ }^{12}$ Here we report the results of whole-exome and targeted sequencing performed to identify mutations that underlie the syndrome in this kindred and in unrelated patients.

\section{METHODS}

\section{STUDY PATIENTS}

Our study included 12 patients with ataxia and hypogonadotropic hypogonadism from eight families. The pedigrees of the index family and four of the other seven families are shown in Figure 1. The patients were referred to the Massachusetts General Hospital for clinical or genetic evaluation between 2000 and 2010. The study was approved by the hospital's human research committee, and written informed consent for all participants was provided by the participant or an authorized representative.

\section{GENETIC ANALYSIS}

We performed exome sequencing with DNA from Patient 3 in the index family. The data sets used for exome analysis in this study were obtained from dbGaP at www.ncbi.nlm.nih.gov/gap through dbGaP accession number phs000475. v1.p1. Candidate genes were sequenced in family members and in unrelated affected persons. Computer algorithms were used to predict the pathogenicity of variants and to identify interactions between candidate genes and genes known to be associated with ataxia or hypogonadotropic hypogonadism. Allele-specific reverse-transcriptase-polymerase-chain-reaction (RT-PCR) assays were performed with RNA from Patients 5, 6, and 7 (see the Methods section in the Supplementary Appendix, available with the full text of this article at NEJM.org).

\section{NEUROPATHOLOGICAL AND ENDOCRINE EVALUATION}

The brain of Patient 2 was obtained within 6 hours after death. Immunohistochemical analysis was performed with the use of antibodies against ubiquitin, tau, and $\alpha$-synuclein. Electron microscopy was performed according to standard procedures. Detailed reproductive endocrine phenotyping was performed in 5 patients, as described in our previous report ${ }^{12}$ and in the Methods section in the Supplementary Appendix.

\section{ZEBRAFISH INVESTIGATIONS}

Morpholino oligonucleotides (MO) for the silencing of zebrafish rnf216 and otud4 were injected either alone or with nonmutant or mutant human messenger RNA (mRNA) encoding RNF216, mRNA encoding OTUD4, or both (see the Methods section in the Supplementary Appendix).

\section{RESULTS}

\section{GENETIC STUdies}

The consanguineous pedigree of the index family (a self-reported Palestinian family) includes three siblings (Patients 1, 2, and 3) with ataxia and hypogonadotropic hypogonadism. Exome sequencing performed with DNA from Patient 3 identified 13 homozygous variants that were rare and predicted to be deleterious (Table S1 in the Supplementary Appendix), 2 of which were also homozygous in the two other affected siblings: RNF216 (NM_207111.3) c.2251C $\rightarrow$ T, p.R751C and OTUD4 (NM_001102653.1) c.998G $\rightarrow$ T, p.G333V; these variants were not identified or were heterozygous in the unaffected family members (Fig. 1). RNF216 encodes an E3 ubiquitin-protein ligase. The R751 residue of RNF216 resides within the second of two domains called "really interesting new gene" (RING) finger domains and is conserved across vertebrates (Fig. S1 in the Supplementary Appendix). The $\mathrm{R} 751 \mathrm{C}$ variant is predicted to be deleterious by four prediction 


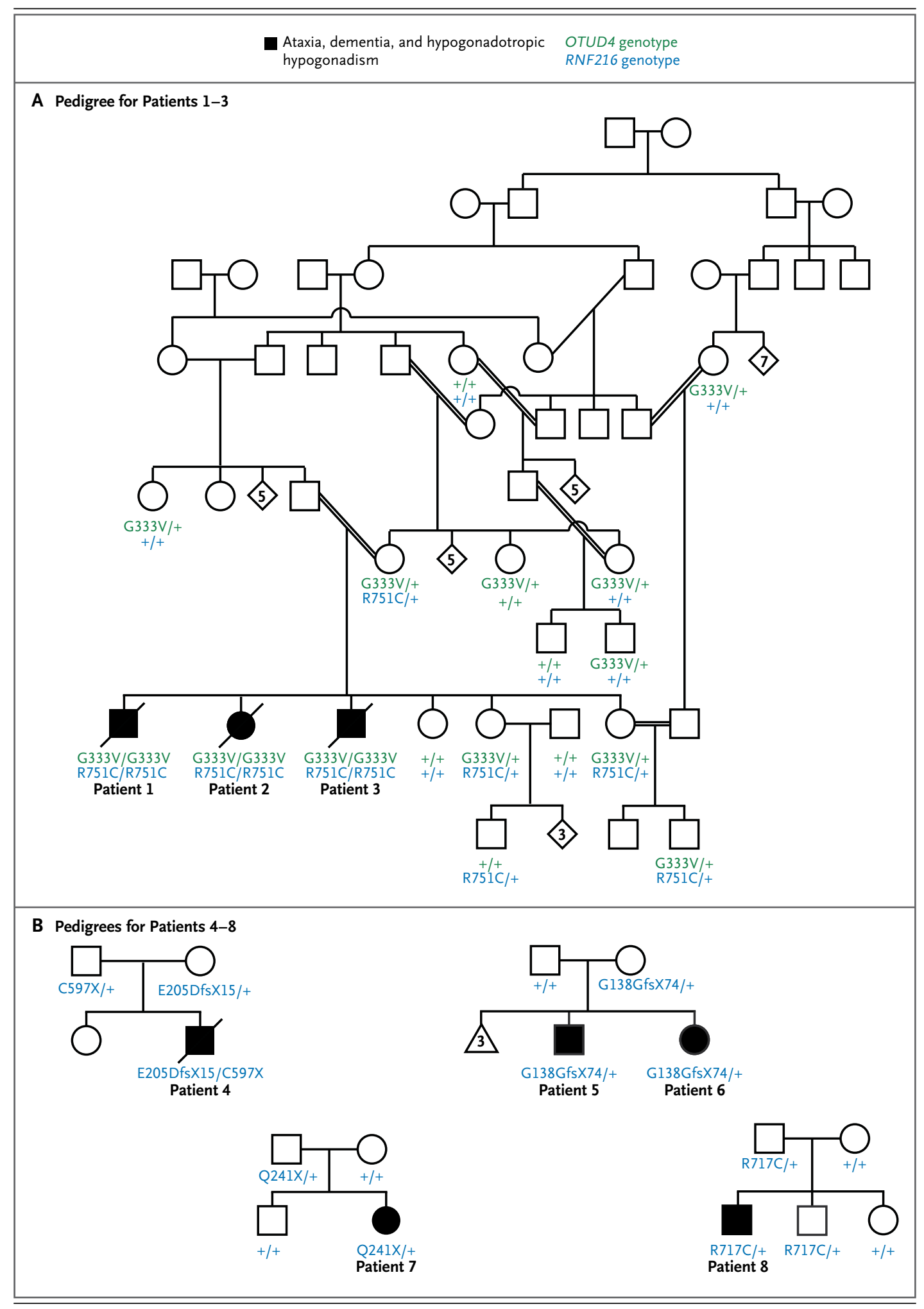

programs and is absent in 13,006 control chro- and in 672 chromosomes from Middle Eastern mosomes from the National Heart, Lung, and persons (including 36 chromosomes from PalesBlood Institute's Exome Sequencing Project (ESP) tinian persons). OTUD4 encodes a deubiquitinase 
Figure 1 (facing page). Segregation of RNF216 and OTUD4 Mutations in the Index Pedigree and Identification of Additional RNF216 Mutations in Unrelated Probands.

The seven-generation pedigree shown in Panel A includes Patients 1, 2, and 3, all of whom presented with ataxia, dementia, and hypogonadotropic hypogonadism and were homozygous for both RNF216 p.R751C and OTUD4 p.G333V. Double lines indicate consanguineous unions. Genotyped, unaffected family members are shown to be either homozygous for the nonmutated alleles (denoted with a + symbol) or heterozygous for one or both changes. The pedigrees shown in Panel B are for the families of additional RNF216 mutation-positive patients (Patients 4 through 8), all of whom presented with ataxia and hypogonadotropic hypogonadism. Squares denote male family members, circles female family members, solid symbols affected family members, slashes deceased family members, diamonds siblings of either sex, the triangle miscarriages, and Arabic numbers the number of siblings or miscarriages.

(Fig. S1 in the Supplementary Appendix). The G333V variant is predicted to be deleterious by three of four prediction programs and is found in 2 of the 13,006 chromosomes from the ESP and in none of the 672 chromosomes from Middle Eastern persons.

Both OTUD4 and RNF216 were sequenced in nine affected persons from seven unrelated families. No rare variants were identified in OTUD4, but mutations in RNF216 were identified in four probands (Fig. 1, and Fig. S1 in the Supplementary Appendix). Patient 4 had compound heterozygous frameshift and nonsense mutations ([c.615_616delGA; p.E205DfsX15] and [c.1791T $\rightarrow$ A; p.C597X]). Three additional heterozygous mutations were identified: c.414delG, p.G138GfsX74 in Patients 5 and 6 (siblings); c.721C $\rightarrow$ T, p.Q241X in Patient 7; and c.2149C $\rightarrow$ T, p.R717C in Patient 8; the missense mutation in Patient 8 was predicted to be deleterious by four prediction programs.

None of these RNF216 variants were present in the ESP, which did identify five other variants considered to be overtly deleterious and nine considered likely to be deleterious. Given this background level of genetic variation, the presence of deleterious mutations in five of eight probands in this study exceeded what would be expected by chance $\left(\mathrm{P}<1 \times 10^{-13}\right.$ by Fisher's exact test). Grail, ${ }^{14}$ DAPPLE, ${ }^{15}$ Endeavour, ${ }^{16}$ InWeb scored network, ${ }^{17}$ and $\mathrm{CNV}$ connect ${ }^{18}$ were used to identify potential connections among RNF216, OTUD4, and genes known to be associated with ataxia or hypogo- nadotropic hypogonadism; no such connections were found. Allele-specific RT-PCR assays failed to identify occult mutations affecting transcription or mRNA stability in the nonmutant alleles of Patients 5, 6, and 7 (Fig. S2 in the Supplementary Appendix).

\section{FUNCTIONAL TESTING}

Zebrafish were used to interrogate the functional consequences and suggestive epistatic interactions of the RNF216 and OTUD4 mutations. ${ }^{19,20}$ The injection of a MO that disrupted the splicing of zebrafish $\mathrm{rnf} 216$ (Fig. S3 in the Supplementary Appendix) caused a reduction in the size of the eye cup and optic tecta and disorganization of the cerebellum, as well as a slight reduction in overall head size (Fig. 2 and 3, and Fig. S4 in the Supplementary Appendix). The tectal phenotype was rescued by the coinjection of human RNF216 mRNA, but the injection of human RNF216 mRNA encoding R751C failed to rescue the phenotype (Fig. 2), suggesting that the mutation affects the function of the encoded protein. The injection of a splice-blocking $\mathrm{MO}$ against zebrafish otud4 (Fig. S3 in the Supplementary Appendix) also induced a reduction in size of the optic tecta and cerebellum (Fig. 3).

Coinjection of both rnf216 and otud4 MOs induced a significant reduction in the size of the optic tecta as compared with the injection of the rnf216 MO alone $(\mathrm{P}<0.001)$ (Fig. 3). Double-MO injection also caused marked disorganization of the cerebellum in more than $60 \%$ of embryos (Fig. 3) and the development of a severe cerebellar phenotype with complete loss of structural integrity in approximately $30 \%$ of embryos, as compared with only 5 to $10 \%$ of embryos injected with either the rnf216 or the otud4 MO alone (data not shown). Furthermore, double-MO injection resulted in marked microphthalmia as compared with modest microphthalmia when either MO injection was used alone (Fig. S4 in the Supplementary Appendix). These phenotypes were specific, since coinjection of nonmutant human RNF216 or OTUD4 mRNA rescued all phenotypes (Fig. 3, and Fig. S4 in the Supplementary Appendix). RNF216 mRNA encoding R751C and OTUD4 mRNA encoding G333V were less effective in rescuing the phenotypes induced by double-MO injection (Fig. 3, and Fig. S4 in the Supplementary Appendix), suggesting not only that these mutant alleles encode functionally deficient proteins but also that epistatic interac- 

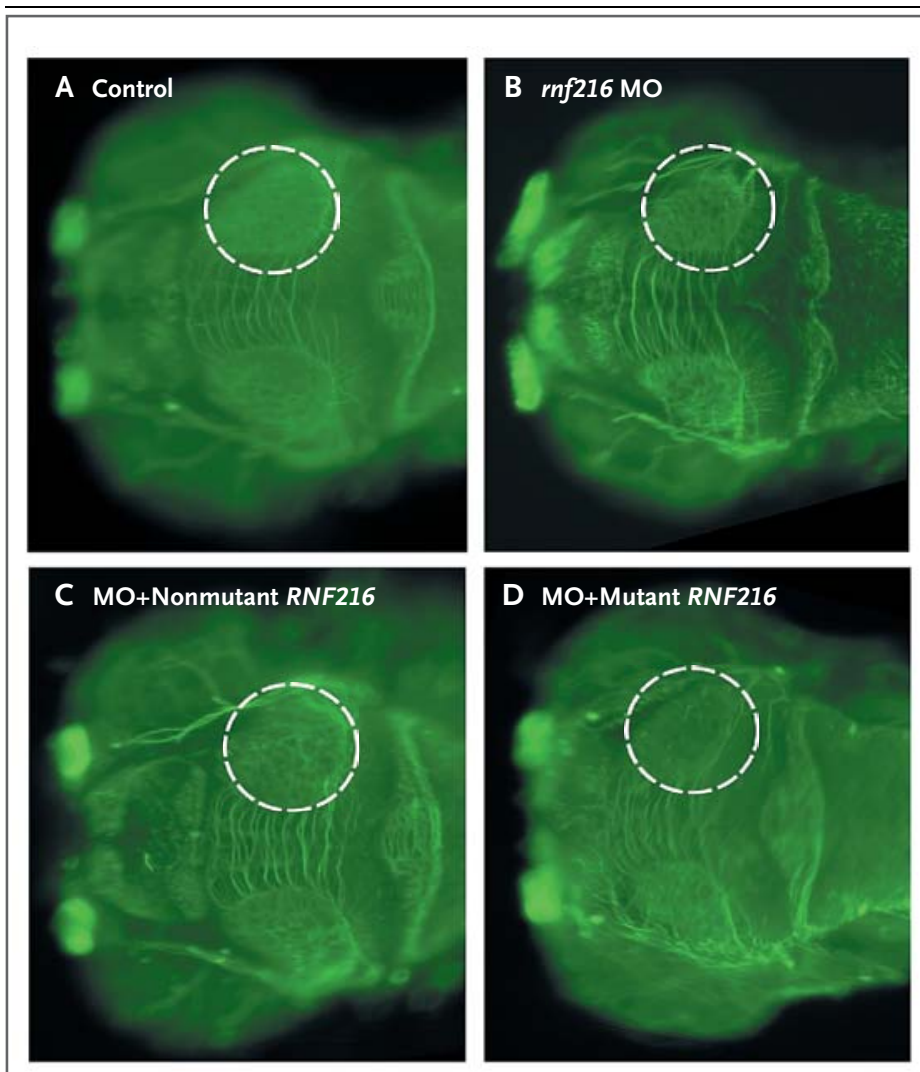

E Size of Optic Tectum

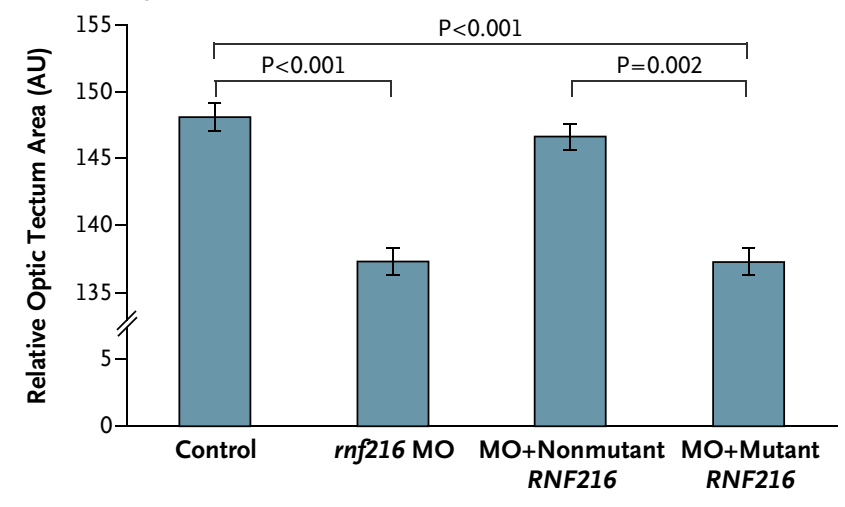

Figure 2. Functional Studies of $r$ nf216 in Zebrafish.

Panels $A$ through $D$ show dorsal views of control zebrafish embryos (Panel A) and embryos injected with $r n f 216$ morpholino oligonucleotides (MO) (Panel B), rnf216 MO plus nonmutant human RNF216 (Panel C), and rnf216 MO plus mutant human RNF216 (with RNF216 carrying the p.R751C mutation identified in the index pedigree) (Panel D) at 3 days after fertilization (staining with an antibody against $\alpha$ acetylated tubulin). The circles outline the area of the optic tectum, the structure on which all measurements were based. The bar graph in Panel E shows the relative size of the optic tectum in control embryos and the embryos injected with $r n f 216 \mathrm{MO}, r n f 216 \mathrm{MO}$ plus nonmutant human RNF216, and $r n f 216 \mathrm{MO}$ plus mutant human RNF216. P values are based on two-tailed t-tests. I bars indicate standard errors. AU denotes arbitrary units. tions between these mutations contribute to the disease phenotype in the index pedigree.

\section{CLINICAL CHARACTERISTICS OF THE STUDY PATIENTS}

Patients 1 through 8, who carried variants in RNF216, had similar clinical histories (Table 1). They presented in adolescence or early adulthood with hypogonadotropic hypogonadism but no other pituitary abnormalities. Dysarthria was the initial neurologic symptom in some patients, but ataxia developed in all patients, leading to wheelchair dependency and to bed confinement for some patients. Dementia was also prominent, with personality changes and memory loss occurring at the onset of the disease and mutism and uncoordinated, purposeless movements during the end stages. Nystagmus was absent. The presentation of Patients 9 through 12, who did not have variants in RNF216, was quite different from that of Patients 1 through 8 (Table 1). Extensive evaluation did not reveal any known causes of ataxia in any of the patients; mitochondrial abnormalities were identified in Patients 7 and 8 (Table S2 in the Supplementary Appendix).

Neuroimaging performed in Patients 1 through 8 revealed striking similarities, with cerebellar and cortical atrophy but no abnormalities of the pituitary gland. The subcortical white matter contained patchy areas of hyperintensity on $\mathrm{T}_{2}$-weighted imaging and fluid-attenuated inversion recovery (FLAIR) imaging (Table 1 and Fig. 4). In Patient 7, these areas of hyperintensity were present approximately 9 years before the onset of neurologic symptoms; the cerebellum appeared normal at that earlier point in time.

\section{NEUROPATHOLOGICAL STUDIES}

The formalin-fixed brain of Patient 2 weighed $940 \mathrm{~g}$ (normal weight, $1300 \mathrm{~g}$ ). The cerebellum and inferior olives were atrophic. Histopathological analysis revealed gliosis and virtually complete loss of inferior olivary neurons, cerebellar Purkinje's cells, and neurons in hippocampal regions CA3 and CA4, whereas neurons were well preserved in regions CA1 and CA2. Ubiquitinimmunoreactive nuclear inclusions were present in 1 to $5 \%$ of the pyramidal neurons in hippocampal regions CA1 and CA2 (Fig. 4) and were also found in granule-cell neurons in the dentate gyrus; these inclusions were not immunoreactive to antibodies against tau or $\alpha$-synuclein (not shown). 
Figure 3. Epistatic Effects of the OTUD4 p.G333V Allele. Panels $A$ through $F$ show dorsal views of control zebrafish embryos (Panel A) and embryos injected with rnf216 MO (morpholino oligonucleotides) (Panel B), otud4 MO (Panel C), double MO (DMO, rnf216 MO plus otud4 MO) (Panel D), double MO plus nonmutant human OTUD4 (Panel E), and double (DMO) plus mutant human OTUD4 (OTUD4 carrying the p.G333V mutation identified in the index pedigree) (Panel F) at 3 days after fertilization (anti- $\alpha$ acetylated tubulin stain). The asterisks indicate the optic tecta that were measured to assess the differences between the conditions being evaluated. The bar graph in Panel $G$ shows the mean relative size of the optic tecta in control embryos and the five groups of injected embryos. I bars indicate standard errors. $P$ values are based on two-tailed t-tests. Panels $\mathrm{H}, \mathrm{I}$, and J show dorsal views of control embryos (Panel $\mathrm{H}$ ) and embryos injected with DMO (Panel I) and DMO plus nonmutant human OTUD4 (Panel $J$ ) at 3 days after fertilization (anti- $\alpha$ acetylated tubulin stain). The rectangles outline the cerebellar area; maximum disorganization is observed in embryos injected only with DMO (Panel I). The bar graph in Panel $\mathrm{K}$ shows the percentage of embryos with cerebellar defects under the conditions being evaluated (as shown in Panels $\mathrm{A}$ through $\mathrm{F}$ and Panels $\mathrm{H}, \mathrm{I}$, and $\mathrm{J}$ ).

On electron microscopy, the intranuclear inclusions appeared as aggregates of fine filaments and granular material (Fig. 4).

\section{REPRODUCTIVE ENDOCRINE STUDIES}

When Patient 6 reached 32 years of age, 1 year after the development of neurologic symptoms, low-amplitude pulses of luteinizing hormone were detected, indicating that GnRH secretion, although present, was diminished (Fig. 5). The administration of pulsatile GnRH for 7 days induced robust increases in levels of gonadotropins and estradiol (Fig. 5) as well as the growth of a dominant ovarian follicle, observed on ultrasonography (not shown). Although the secretion of luteinizing hormone increased in response to the administration of $\mathrm{GnRH}$, the typical peaked pattern of luteinizing hormone pulses ${ }^{21}$ was not seen, which suggested a degree of pituitary dysfunction. Indeed, the patient's pituitary responsiveness waned over time, with a diminished response to GnRH on day 1 of treatment 15 months after the initial endocrine study (Fig. 5).

In Patient 8, in whom endocrine function was initially assessed before the onset of neurologic symptoms, there was an absence of endogenous pulsatile luteinizing hormone secretion (Fig. 5).
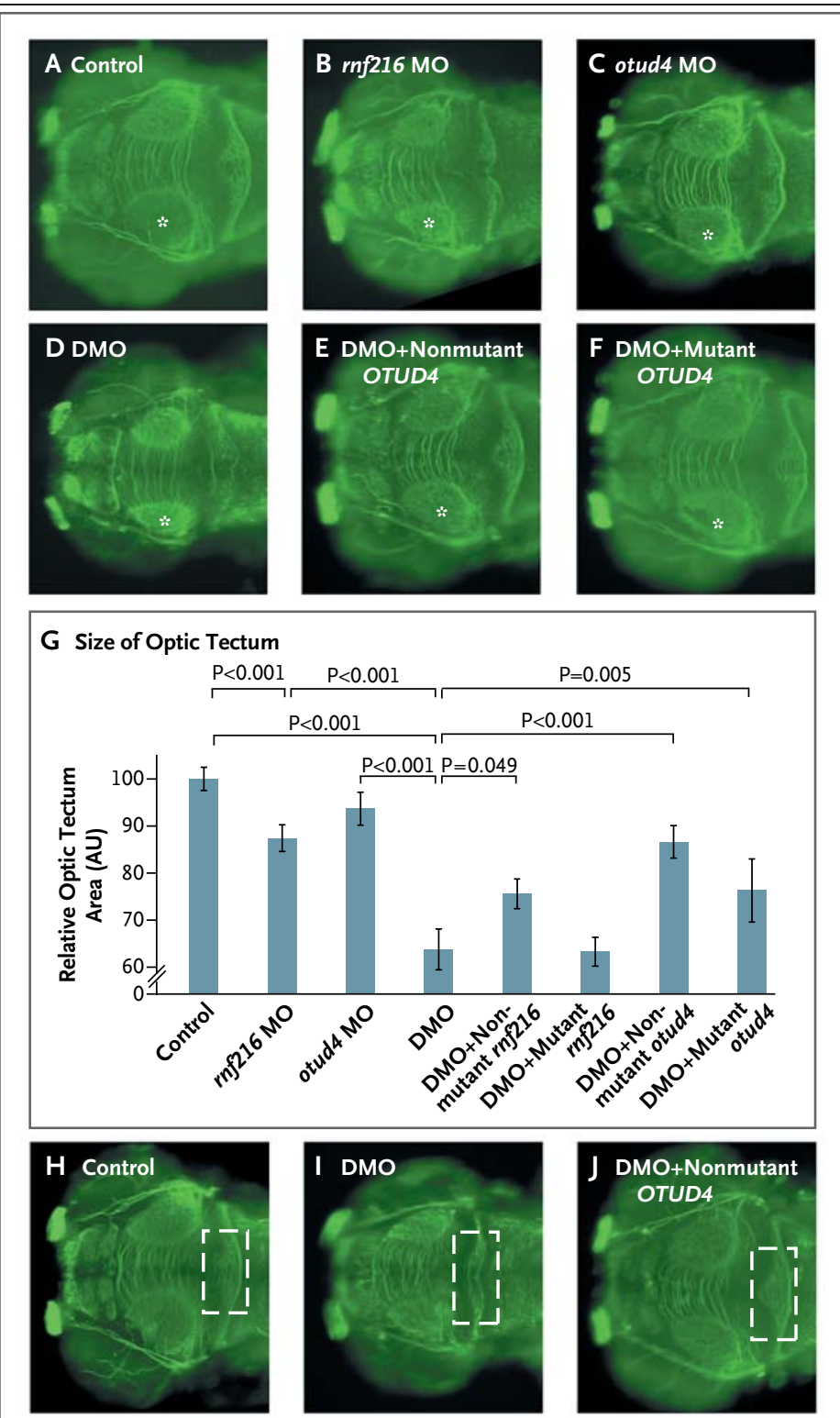

K Percentage of Embryos with Defects

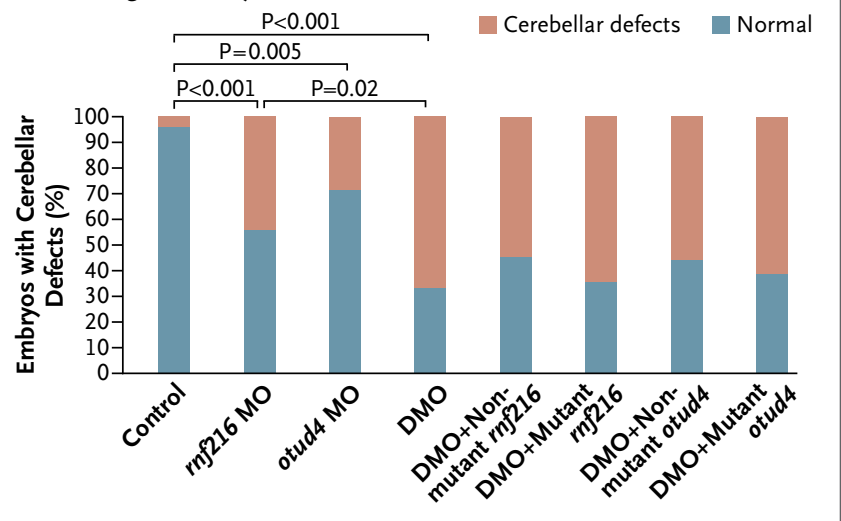




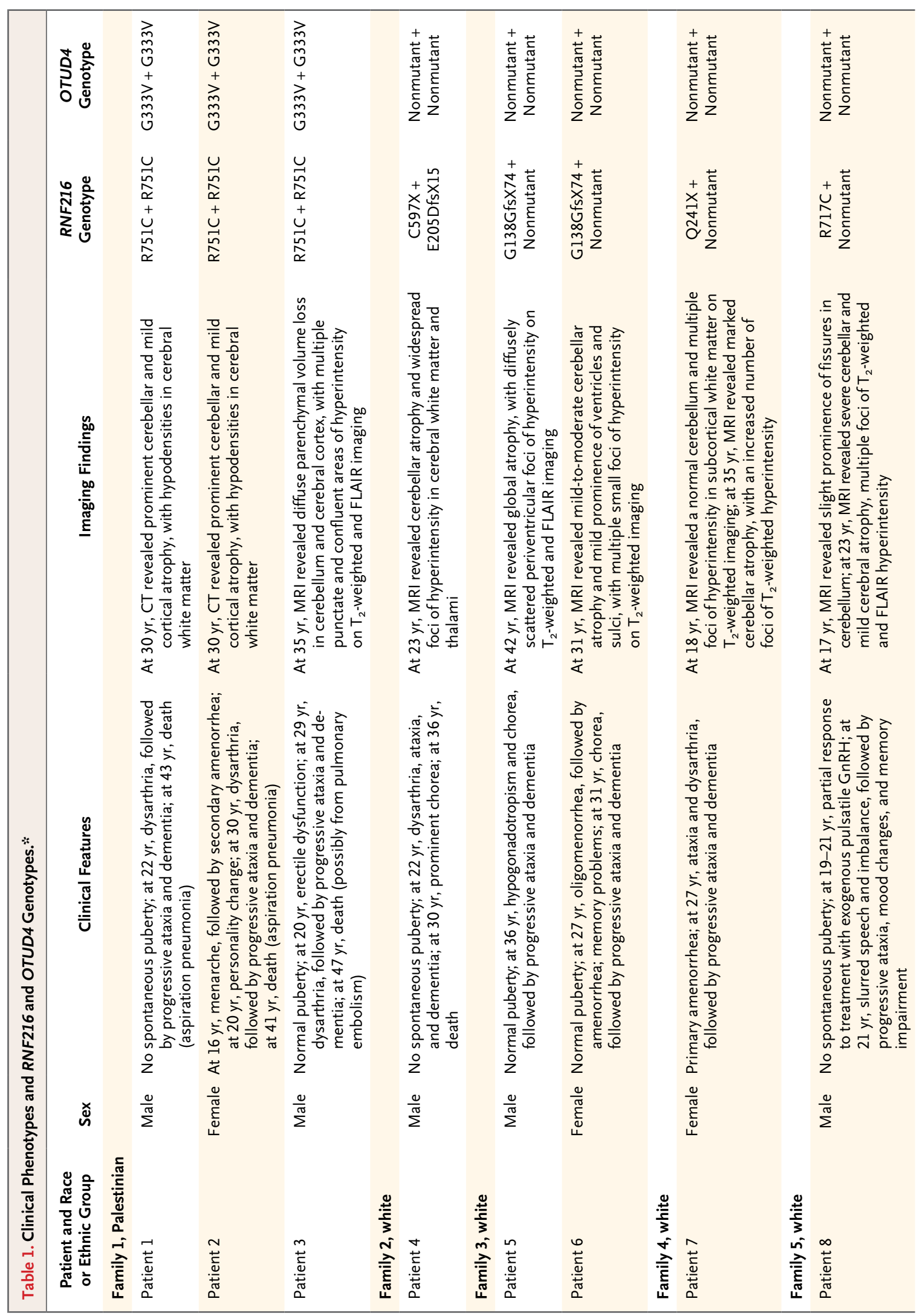




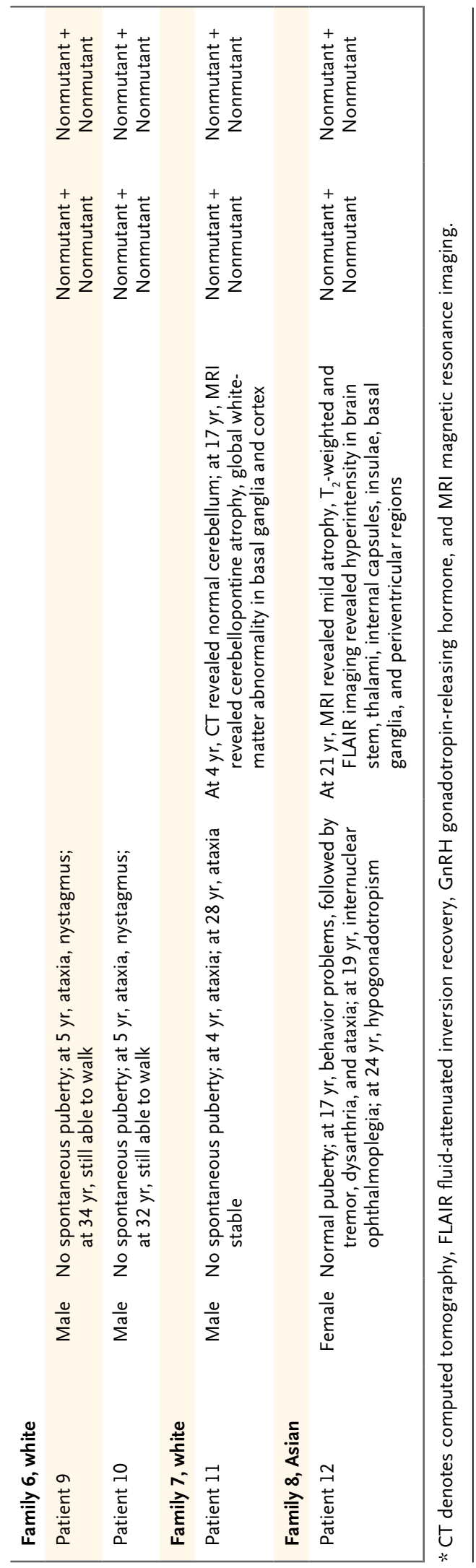

After escalating doses of exogenous GnRH (from $25 \mathrm{ng}$ per kilogram of body weight to $600 \mathrm{ng}$ per kilogram every 2 hours), the patient's testicular volume increased from $2 \mathrm{ml}$ to $8 \mathrm{ml}$ but did not increase further, despite these very high doses. Although his pituitary response to exogenous treatment with GnRH was impaired (Fig. 5), direct gonadal stimulation with human chorionic gonadotropin normalized the testosterone level, at $459 \mathrm{ng}$ per deciliter (15.9 $\mathrm{nmol}$ per liter).

Pituitary responsiveness to GnRH was lost in patients late in the course of their disease. In Patients 1 and 2, who were bedridden when pituitary responsiveness was assessed, there was no detectable luteinizing hormone secretion and no measurable change in the gonadotropin level in response to the administration of pulsatile exogenous GnRH (Fig. 5). ${ }^{12}$

\section{DISCUSSION}

The underpinnings for the association of ataxia with hypogonadotropic hypogonadism have eluded investigators for more than a century. This report shows that ataxia with hypogonadotropic hypogonadism can be caused by mutations in RNF216 either singly or in combination with mutations in OTUD4. Both RNF216 and OTUD4 encode proteins that regulate ubiquitination, indicating that abnormalities in this fundamental cellular process can have pathologic effects on the cerebellum and hippocampus, the cerebral white matter, and the hypothalamic and pituitary components of the reproductive endocrine cascade.

The compound heterozygous termination mutations in RNF216 in Patient 4 firmly implicate RNF216 as a causative gene for this syndrome. Heterozygous RNF216 mutations were found in Patients 5 through 8 but did not cause disease in their parents. Oligogenicity offers a parsimonious explanation for these observed patterns. Oligogenic inheritance has been described in the Bardet-Biedl and Bartter syndromes, Hirschsprung's disease, and isolated hypogonadotropic hypogonadism. ${ }^{19,22-27}$ In such an oligogenic model, RNF216 mutations can act with mutations at other genetic loci to cause disease.

Indeed, the phenotype in the index pedigree appears to have been caused by the interaction of hypomorphic mutations in RNF216 and OTUD4. Inhibition of either RNF216 or OTUD4 in zebrafish resulted in cerebellar hypoplasia, microph- 

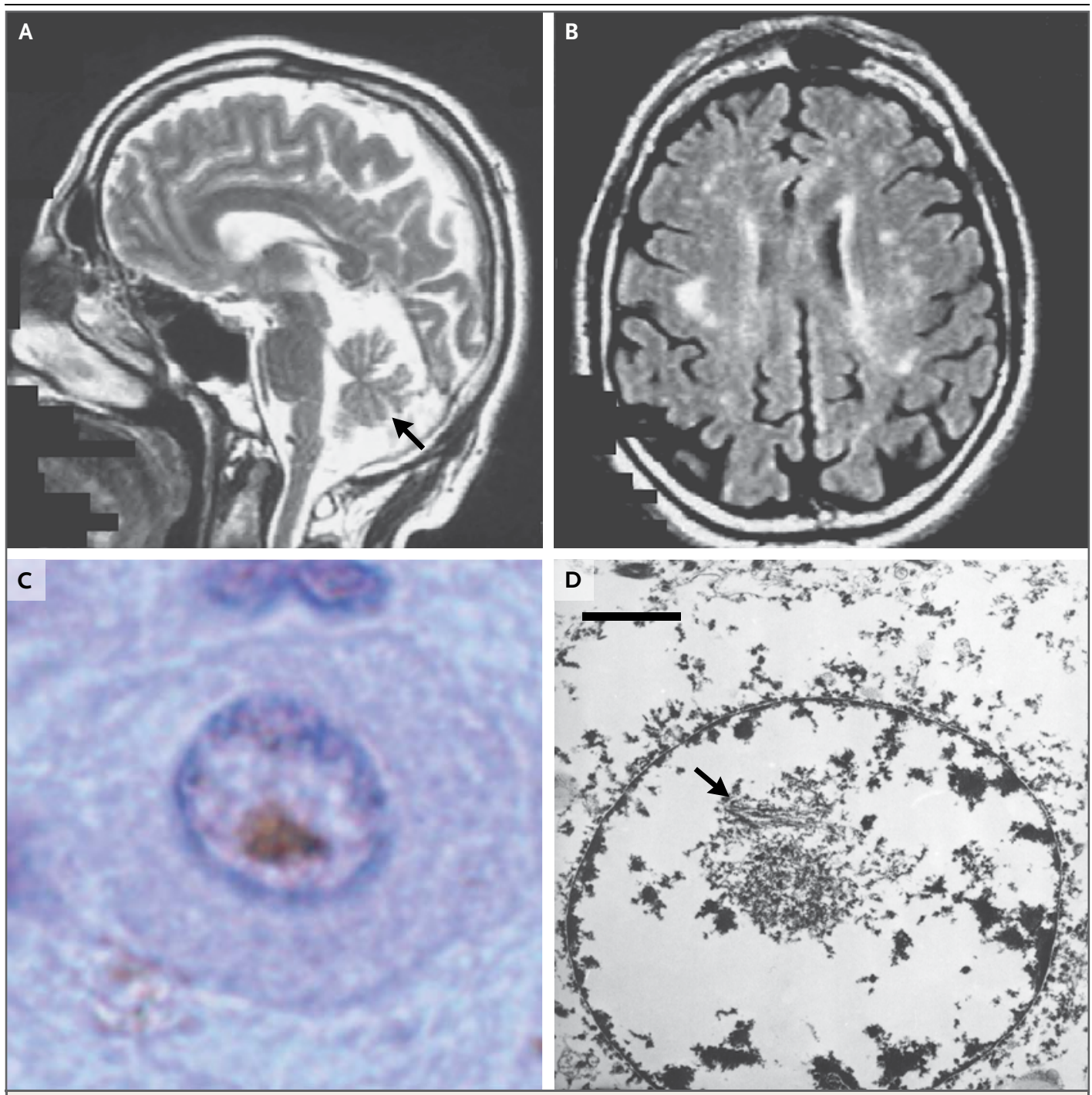

Figure 4. Neuroradiologic and Neuropathological Findings.

Panel A shows a sagittal $\mathrm{T}_{2}$-weighted magnetic resonance imaging scan of the brain in Patient 3 . Diffuse cerebellar atrophy (arrow) and cortical atrophy can be seen. Panel B shows a transverse image obtained with fluid-attenuated inversion recovery imaging, revealing multiple distinct and confluent foci of hyperintensity in the white matter. In Panel C, immunohistochemical analysis of a hippocampal brain section from Patient 2 shows a neuronal intranuclear inclusion with immunoreactivity (brown) to an antibody against ubiquitin, counterstained with hematoxylin and eosin. An electron micrograph of the hippocampal neurons, in Panel D, also shows an intranuclear inclusion, which consists of aggregates of granular material and fine filaments, 10 to $15 \mathrm{~nm}$ in diameter (arrow), that are for the most part randomly oriented. The scale bar corresponds to $1 \mu \mathrm{m}$.

thalmia, and small optic tecta. The concordance of these phenotypes suggests that RNF216 and OTUD4 operate in the same pathway. This possibility is bolstered by the observation of epistatic interactions between RNF216 and OTUD4, with simultaneous knockdown of both genes resulting in more severe phenotypes. Taken together, these findings support a digenic model in which the OTUD4 mutation, in conjunction with the RNF216 mutation, played an essential role in causing disease in the index pedigree. By extension, in Patients 5 through 8 , it is possible that mutations in other, currently unidentified loci may have acted in conjunction with the heterozygous mutations in RNF216 to cause disease. Oligogenicity is likely to be increasingly recognized as methods for detecting this genetic architecture, such as exome sequencing, are more widely adopted. 


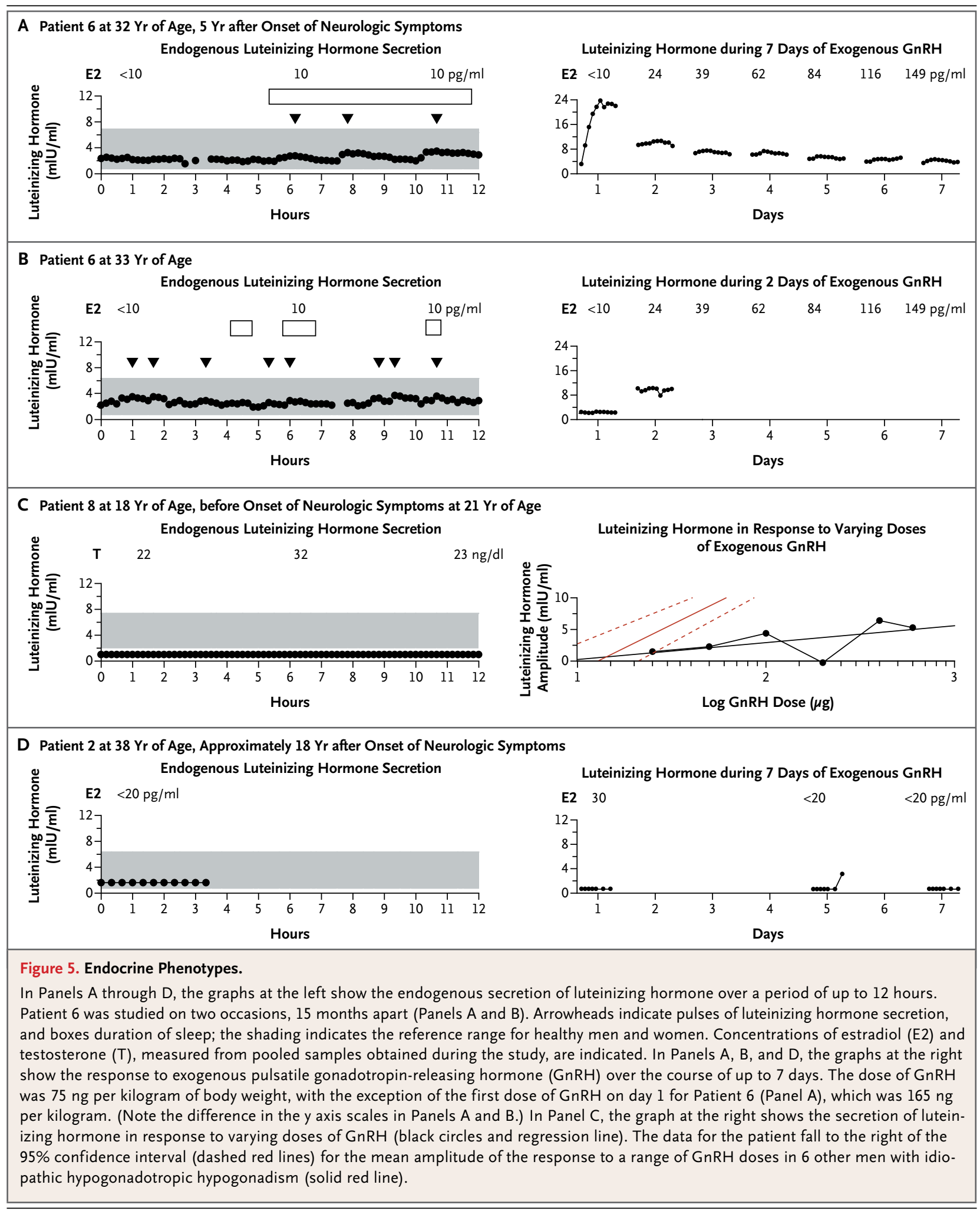

RNF216 encodes an E3 ubiquitin ligase that Known targets of RNF216 include upstream attaches ubiquitin to protein substrates, mark- activators of nuclear factor $\kappa \mathrm{B}$ signaling, which ing them for proteasome-mediated degradation. regulates diverse cellular processes. ${ }^{28-31}$ RNF216 
is structurally similar to parkin, an E3 ubiquitin ligase that is mutated in a recessive form of Parkinson's disease. ${ }^{32-34}$ The finding of neuronal intranuclear inclusions in Patient 2 may indicate that RNF216-associated neurodegeneration has similarities not only with Parkinson's disease but also with other neurodegenerative disorders in which protein aggregates are found, such as Huntington's disease and Alzheimer's disease. ${ }^{35}$

OTUD4 encodes a deubiquitinating enzyme. Deubiquitinases allow target proteins and ubiquitin itself to be recycled and often function in partnership with specific E3 ligases. For example, the deubiquitinase ataxin-3 counteracts the ability of parkin to ubiquitinate itself. ${ }^{36}$ On the basis of this and other examples, ${ }^{37}$ OTUD4 and RNF216 may be similarly linked in a coregulatory partnership.

The progressive and debilitating dementia observed in the patients with RNF216 mutations (Patients 1 through 8) distinguishes them from the other patients with ataxia and hypogonadotropic hypogonadism. Furthermore, we observed changes in cerebral white matter in all the patients with RNF216-associated neurodegeneration, suggesting that such changes may constitute a consistent feature of this syndrome. None of these patients had oculomotor abnormalities such as the nystagmus and ophthalmoplegia seen in Patients 9, 10, and 12, who did not have RNF216 mutations.

The patients with RNF216-associated neurodegeneration had dysfunction at multiple levels of the reproductive endocrine axis. In Patients 6 and 8 , reproductive function was restored with extended GnRH treatment, which suggests that hypothalamic GnRH deficiency was the primary cause of their reproductive endocrine dysfunction. However, these two patients also appeared to have an element of pituitary dysfunction, given the diminishing responses to GnRH over time in Patient 6 and the observation of a right-shifted dose-response curve in Patient 8. In Patients 1 and 2 , who were evaluated late in the course of their disease, the complete absence of response after 7 days of treatment with GnRH may represent progression of this pituitary dysfunction. The basis for the selective vulnerability of particular neuronal and pituitary cell types is currently unexplained.

In conclusion, we have identified loss-of-function mutations in RNF216 that cause a syndrome of ataxia, dementia, and hypogonadotropic hypogonadism. Genetic and in vivo evidence suggests that mutations affecting RNF216, an E3 ubiquitin ligase, and OTUD4, a deubiquitinase, can synergize to cause this syndrome, reinforcing the notion that the mutational load within biologic pathways can drive disease manifestation. ${ }^{20}$ Taken together, these data highlight a hitherto unknown role of the ubiquitination system in disorders of combined neurodegeneration and reproductive dysfunction. More broadly, our findings show the value of combining individual whole-exome sequencing with in vivo functional studies to identify disease-causing gene mutations and epistatic interactions.

The views expressed in this article are those of the authors and do not necessarily represent the official views of Harvard Catalyst, Harvard University and its affiliated academic health care centers, or the National Institutes of Health.

Supported by grants from the Eunice K. Shriver National Institute for Child Health and Human Development (K24 HD067388, R01 HD043341, R01 HD042601, and U54 HD028138); from the National Institute of Diabetes and Digestive and Kidney Diseases (P50 DK096415); by Harvard Catalyst and the Harvard Clinical and Translational Science Center (funded by the National Center for Research Resources and the National Center for Advancing Translational Sciences [UL1TR025758 and M01RR01066] and Harvard University and its affiliated academic health care centers); by a grant from the National Human Genome Research Institute to the Broad Institute (U54 HG003967); and by grants from the National Heart, Lung, and Blood Institute for the Exome Sequencing Project (HL102923, HL102924, HL102925, HL102926, and HL103010). Dr. Chan is the recipient of a Charles A. King Trust postdoctoral fellowship and a Career Development Award from Boston Children's Hospital.

Disclosure forms provided by the authors are available with the full text of this article at NEJM.org.

We thank the patients and their families for their cooperation in these studies; Dr. Fawzi al-Hammouri and the physicians and staff of the Specialty Hospital, Amman, Jordan, for assisting with clinical care for Patients 1, 2, and 3; Dr. Momen al-Hadidi and the staff of the Forensic Medicine Department at Al-Basheer Government Hospital, Amman, Jordan, for performing the autopsy and obtaining the brain after the death of Patient 2; Drs. Daniel Metzger, Susan Ratzan, Meriel McEntagart, and Sandra Sirrs for patient referrals; Dr. J. Michael Andresen for performing the initial linkage analysis; Drs. Christopher Walsh and Timothy Yu at Boston Children's Hospital for sharing data from the Middle Eastern control patients; Carlotta Fitch and Dr. Kathy Newell for assisting with the neuropathological analyses; Dr. Omar Abu Hijleh for providing the early endocrine history of Patients 1 and 2; the staff of the Massachusetts General Hospital (MGH) Clinical Research Center for assisting with clinical protocols; members of the MGH Reproductive Endocrine Unit; and Eric Lander and the Broad Institute for generating high-quality sequence data. 
1. Sailer A, Houlden H. Recent advances in the genetics of cerebellar ataxias. Curr Neurol Neurosci Rep 2012;12:227-36.

2. Holmes G. A form of familial degeneration of the cerebellum. Brain 1908;30: 466-89.

3. Volpé R, Metzler WS, Johnston MW. Familial hypogonadotrophic eunuchoidism with cerebellar ataxia. J Clin Endocrinol Metab 1963;23:107-15.

4. Lowenthal A, Bekaert J, Van Dessel F, van Hauwaert J. Familial cerebellar ataxia with hypogonadism. J Neurol 1979;222: 75-80.

5. Berciano J, Amado JA, Freijanes J, Rebollo M, Vaquero A. Familial cerebellar ataxia and hypogonadotropic hypogonadism: evidence for hypothalamic LHRH deficiency. J Neurol Neurosurg Psychiatry 1982;45:747-51. [Erratum, J Neurol Neurosurg Psychiatry 1983;46:472.]

6. Fok AC, Wong MC, Cheah JS. Syndrome of cerebellar ataxia and hypogonadotrophic hypogonadism: evidence for pituitary gonadotrophin deficiency. J Neurol Neurosurg Psychiatry 1989;52:407-9.

7. Abs R, Van Vleymen E, Parizel PM, Van Acker K, Martin M, Martin JJ. Congenital cerebellar hypoplasia and hypogonadotropic hypogonadism. J Neurol Sci 1990;98:259-65.

8. De Michele G, Filla A, D'Armiento FP et al. Cerebellar ataxia and hypogonadism: a clinicopathological report. Clin Neurol Neurosurg 1990;92:67-70.

9. De Michele G, Filla A, Striano S, Rimoldi M, Campanella G. Heterogeneous findings in four cases of cerebellar ataxia associated with hypogonadism (Holmes' type ataxia). Clin Neurol Neurosurg 1993;95:23-8.

10. Quinton R, Barnett P, Coskeran P, Bouloux PM. Gordon Holmes spinocerebellar ataxia: a gonadotrophin deficiency syndrome resistant to treatment with pulsatile gonadotrophin-releasing hormone. Clin Endocrinol (Oxf) 1999;51:525-9.

11. Vielhaber S, Ebert AD, Feistner $H$, Herrmann M. Frontal-executive dysfunction in early onset cerebellar ataxia of Holmes' type. Clin Neurol Neurosurg 2000;102:102-5.

12. Seminara SB, Acierno JS Jr, Abdulwahid NA, Crowley WF Jr, Margolin DH. Hypogonadotropic hypogonadism and cerebellar ataxia: detailed phenotypic characterization of a large, extended kindred. J Clin Endocrinol Metab 2002;87: 1607-12.

13. Balasubramanian R, Crowley WF Jr.
Isolated GnRH deficiency: a disease model serving as a unique prism into the systems biology of the GnRH neuronal network. Mol Cell Endocrinol 2011;346: 4-12.

14. Raychaudhuri S, Plenge RM, Rossin EJ, et al. Identifying relationships among genomic disease regions: predicting genes at pathogenic SNP associations and rare deletions. PLoS Genet 2009;5(6):e1000534. 15. Rossin EJ, Lage K, Raychaudhuri S, et al. Proteins encoded in genomic regions associated with immune-mediated disease physically interact and suggest underlying biology. PLoS Genet 2011;7(1): e1001273.

16. Aerts S, Lambrechts D, Maity S, et al. Gene prioritization through genomic data fusion. Nat Biotechnol 2006;24:537-44. [Erratum, Nat Biotechnol 2006;24:719.]

17. Lage $\mathrm{K}, \mathrm{M} \phi$ llgård $\mathrm{K}$, Greenway $\mathrm{S}$, et al. Dissecting spatio-temporal protein networks driving human heart development and related disorders. Mol Syst Biol 2010; 6:381.

18. Longoni M, Lage K, Russell MK, et al. Congenital diaphragmatic hernia interval on chromosome 8 p23.1 characterized by genetics and protein interaction networks. Am J Med Genet A 2012;158A:3148-58.

19. de Pontual L, Zaghloul NA, Thomas S, et al. Epistasis between RET and BBS mutations modulates enteric innervation and causes syndromic Hirschsprung disease. Proc Natl Acad Sci U S A 2009;106: 13921-6.

20. Zaghloul NA, Katsanis N. Zebrafish assays of ciliopathies. Methods Cell Biol 2011;105:257-72.

21. Lavoie HB, Martin KA, Taylor E, Crowley WF, Hall JE. Exaggerated free alphasubunit levels during pulsatile gonadotropin-releasing hormone replacement in women with idiopathic hypogonadotropic hypogonadism. J Clin Endocrinol Metab 1998;83:241-7.

22. Katsanis N, Ansley SJ, Badano JL, et al. Triallelic inheritance in Bardet-Biedl syndrome, a Mendelian recessive disorder. Science 2001;293:2256-9.

23. Katsanis N, Eichers ER, Ansley SJ, et al. BBS4 is a minor contributor to BardetBiedl syndrome and may also participate in triallelic inheritance. Am J Hum Genet 2002;71:22-9.

24. Badano JL, Leitch CC, Ansley SJ, et al. Dissection of epistasis in oligogenic BardetBiedl syndrome. Nature 2006;439:32630.

25. Schlingmann KP, Konrad M, Jeck N, et al. Salt wasting and deafness resulting from mutations in two chloride channels. N Engl J Med 2004;350:1314-9.

26. Nozu K, Inagaki T, Fu XJ, et al. Molecular analysis of digenic inheritance in Bartter syndrome with sensorineural deafness. J Med Genet 2008;45:182-6.

27. Sykiotis GP, Plummer L, Hughes VA, et al. Oligogenic basis of isolated gonadotropin-releasing hormone deficiency. Proc Natl Acad Sci U S A 2010;107:15140-4.

28. Chen D, Li X, Zhai Z, Shu HB. A novel zinc finger protein interacts with receptor-interacting protein (RIP) and inhibits tumor necrosis factor (TNF)- and IL1induced NF- $\kappa \mathrm{B}$ activation. J Biol Chem 2002;277:15985-91.

29. Fearns C, Pan Q, Mathison JC, Chuang TH. Triad3A regulates ubiquitination and proteasomal degradation of RIP1 following disruption of Hsp90 binding. J Biol Chem 2006;281:34592-600.

30. Miah SM, Purdy AK, Rodin NB, et al. Ubiquitylation of an internalized killer cell Ig-like receptor by Triad3A disrupts sustained NF- $\kappa$ B signaling. J Immunol 2011; 186:2959-69.

31. Chuang TH, Ulevitch RJ. Triad3A, an E3 ubiquitin-protein ligase regulating Tolllike receptors. Nat Immunol 2004;5:495502. [Erratum, Nat Immunol 2004;5:968.] 32. Pilsl A, Winklhofer KF. Parkin, PINK1 and mitochondrial integrity: emerging concepts of mitochondrial dysfunction in Parkinson's disease. Acta Neuropathol 2012;123:173-88.

33. Kumar P, Pradhan K, Karunya R, Ambasta RK, Querfurth HW. Cross-functional E3 ligases Parkin and C-terminus Hsp70-interacting protein in neurodegenerative disorders. J Neurochem 2012;120: 350-70.

34. Corti O, Lesage S, Brice A. What genetics tells us about the causes and mechanisms of Parkinson's disease. Physiol Rev 2011;91:1161-218.

35. Saxena S, Caroni P. Selective neuronal vulnerability in neurodegenerative diseases: from stressor thresholds to degeneration. Neuron 2011;71:35-48.

36. Durcan TM, Kontogiannea M, Bedard N, Wing SS, Fon EA. Ataxin-3 deubiquitination is coupled to Parkin ubiquitination via E2 ubiquitin-conjugating enzyme. J Biol Chem 2012;287:531-41.

37. Nakada S, Tai I, Panier S, et al. Noncanonical inhibition of DNA damagedependent ubiquitination by OTUB1. Nature 2010;466:941-6.

Copyright (c) 2013 Massachusetts Medical Society.

RECEIVE IMMEDIATE NOTIFICATION WHEN AN ARTICLE IS PUBLISHED ONLINE FIRST

To be notified by e-mail when Journal articles are published Online First, sign up at NEJM.org. 